\title{
A systematic review of evidence-based practices for clinical education and health care delivery in the clinical teaching unit
}

\author{
Brandon Tang MD MSc, Ryan Sandarage BSc, Jocelyn Chai MD, Kristin Anne Dawson MD, Katrina Rose Dutkiewicz MD, \\ Stephan Saad MD, Vanessa Kitchin MI, Rose Hatala MD MSc, lain McCormick MD, Barry Kassen MD
}

Cite as: CMAJ 2022 February 14;194:E186-94. doi: 10.1503/cmaj.202400

\begin{abstract}
Background: The clinical teaching unit is a widespread clinical training model that requires reform to prepare physicians for practice in the 21st century. In this systematic review, we aimed to identify evidence-based practices in internal medicine clinical teaching units that contribute to improved clinical education and health care delivery.
\end{abstract}

Methods: We searched several databases from 1993 until Apr. 5, 2021, to identify published studies in inpatient clinical teaching units that involved medical trainees and reported outcomes related to trainee education or health care delivery. We identified emergent themes using a narrative approach and determined confidence in review findings using the Grading of Recommendations Assessment, Development and Evaluation Confidence in the Evidence from Reviews of Qualitative Research (GRADECERQual) methodology.

Results: We included 107 studies of internal medicine clinical teaching units, of which 93 (87\%) were conducted in North America. Surveys ( $n=31,29 \%)$, trials $(n=17,16 \%)$ and narrative studies ( $n=15,14 \%$ ) were the most prevalent study designs. Practices identified as contributing to improved clinical education or health care delivery included purposeful rounding (high confidence), bedside rounding (moderate confidence), resource stewardship interventions (high confidence), interprofessional rounds (moderate confidence), geographic wards (moderate confidence), allocating more trainee time to patient care or educational activities (moderate confidence), "drip" continuous models of admission (moderate confidence), limiting duty hours (moderate confidence) and limiting clinical workload (moderate confidence).

Interpretation: In this review, we identified several evidence-based practices that may contribute to improved educational and health care outcomes in clinical teaching unit settings. These findings may offer guidance for policies, resource allocation and staffing of teaching hospitals.
M edical education in North America is largely based on an educational model that is now more than a century old. ${ }^{1}$ To keep pace with changing social, economic and health system circumstances, substantial educational reform is necessary to prepare physicians for practice in the 21 st century. Over the past decade, numerous national organizations, including the Association of Faculties of Medicine in Canada and the American Medical Association, have echoed this call to action. ${ }^{1,2}$

The clinical teaching unit (CTU) provides a joint model of undergraduate and postgraduate clinical education in which trainees contribute to direct patient care, with graded levels of responsibility reflective of their level of training. ${ }^{3}$ The CTU describes an approach to delivering learner education and patient care in parallel, and since its inception in Canada in 1962, this model of care has become almost ubiquitous across clinical specialties in many countries. Although the use of CTUs is a common approach to organizing an inpatient teaching service, the implementation of CTUs is highly variable with regard to elements such as the number of learners and patients, and regionalization to specific hospital wards. Moreover, a systematic evaluation of practices that maximize the CTU's effectiveness has not been conducted, meaning evidence to inform CTU design is lacking.

The purpose of this systematic review was to identify practices in internal medicine CTUs that contribute to improved clinical education for resident physicians and medical students, as well as to health care delivery. 


\section{Methods}

\section{Search strategy and study selection}

We conducted a qualitative systematic review of primary research studies of clinical teaching units, in accordance with the Preferred Reporting Items for Systematic Review and MetaAnalyses (PRISMA) publication standards. ${ }^{4}$ We searched the MEDLINE, Embase, Cochrane, CINAHL, PsycINFO, Education Resources Information Center (ERIC), ProQuest and University of British Columbia CiRcle databases for articles published in English or French from 1993 to Apr. 5, 2021. We identified additional articles by screening references of studies meeting inclusion criteria for relevant sources, along with other studies that were identified ad hoc. Detailed search strategies are provided in Appendix 1, Section 1, available at www.cmaj.ca/ lookup/doi/10.1503/cmaj.202400/tab-related-content. We included studies if they were conducted in an inpatient clinical teaching unit that involved resident physicians, medical students or both, and if they described outcomes related to trainee education or health care delivery. Two reviewers independently screened abstracts and full-text articles for eligibility, with any discrepancies resolved through discussion with a third reviewer.

\section{Data extraction and quality assessment}

Two reviewers (B.T., R.S., J.C., K.A.D., K.R.D., S.S.) independently extracted data from each included study using a standardized, pre-piloted form. ${ }^{5}$ Two reviewers (B.T., R.S., J.C., K.A.D., K.R.D.) independently assessed methodological quality for all studies, using the Medical Education Research Study Quality Instrument (MERSQI) ${ }^{6}$ for quantitative studies and the Consolidated Criteria for Reporting Qualitative Research (COREQ) instrument ${ }^{7}$ for qualitative studies. Scoring discrepancies were resolved through discussion with a third reviewer (R.S., B.T.).

\section{Data analysis}

We employed a narrative approach to evidence synthesis, with particular attention to design practices, proposed mechanisms and outcomes identified for each study. We discussed emergent themes until consensus was reached. We assessed the confidence in each review finding using the Grading of Recommendations Assessment, Development and Evaluation Confidence in the Evidence from Reviews of Qualitative Research (GRADECERQual) approach, ${ }^{8}$ which is conceptually similar to other GRADE tools, but is employed in qualitative evidence syntheses. ${ }^{9}$ This approach collectively assesses the strength of evidence contributing to each review finding using 4 criteria, namely methodological limitations, coherence, adequacy and relevance. We categorized findings according to the Star Model, an evidence-based management tool for health system design, which divides complex systems into their interdependent subsystems (i.e., strategy, structure, human resources, incentives, information and decision-making and culture) to facilitate analysis and policy reform. ${ }^{10}$

Additional details of our systematic review methods are provided in Appendix 1, Section 2.

\section{Results}

Our review process is summarized in Figure 1. In total, we considered 2464 studies after removing duplicates; 358 articles underwent full-text review, of which 262 articles met inclusion criteria. Given the range of clinical specialties represented in included studies (Appendix 1, Section 3, Supplementary Table 1), as determined a priori, ${ }^{5}$ we limited data analysis to studies from general internal medicine CTUs to limit contextual variability. Therefore, we included 107 studies in our analysis, which are summarized in Table 1 and described in detail in Appendix 1 , Section 3, Supplementary Table 2.

Most studies ( $n=93,87 \%)$ were conducted in North America. Surveys $(n=31,29 \%)$, trials $(n=17,16 \%)$ and narrative studies $(n=15,14 \%)$ were the most prevalent study designs. Most studies included residents and students $(n=75,70 \%)$, practising physicians ( $n=47,44 \%)$, patients $(n=33,31 \%)$, and entire inpatient wards $(n=21,20 \%)$. We assessed $84(78 \%)$ as either high or medium quality. Among all analyzed studies, 34 (32\%) evaluated educational outcomes, $31(29 \%)$ evaluated patient care outcomes and 42 (39\%) evaluated both types of outcomes (Table 1). Although all subsystems of the Star Model were represented in the analysis, the emergent themes were clustered within the strategy, structure and human resources subsystems. An overview of key findings from this review and the assessment of confidence in each finding are reported in Table 2, Table 3 and Table 4. Comprehensive results from the GRADE-CERQual assessment are reported in Appendix 1, Section 3, Supplementary Table 3.

\section{Strategies to improve learner education and patient care}

\section{Purposeful rounding}

Purposeful rounding, in which the order that patients should be seen is explicitly discussed, was explored in 6 studies, including 5 medium- or high-quality studies (Table 2). ${ }^{11-16}$ This approach was frequently incorporated in broader redesigns of the rounding process, ${ }^{11-13}$ with 2 high-quality studies reporting a shorter duration of rounds after intervention. ${ }^{12,14}$ Given the diverse terminology used to describe different rounding approaches, a proposed standardized nomenclature is suggested in Appendix 1, Section 3, Supplementary Table 4. Although the specific impact of purposeful rounding is challenging to delineate in multiinterventional studies, a high-quality observational study of 11 inpatient teams found that teams employing purposeful rounding had a shorter mean duration of rounds $(92.8 \mathrm{v}$. $119.0 \mathrm{~min}$ ), reduced average length of stay for patients (4.6 v. $5.7 \mathrm{~d}$ ) and lower complication rates ( 0.2 v. 0.5 per patient per day)..$^{15}$ Purposeful rounding was encouraged through prerounds or morning huddles, in which sick patients and potential discharges were typically prioritized to be seen first. ${ }^{11,13,14,16}$

\section{Bedside rounds}

Bedside rounding, in which the team meets with the patient at the bedside and discussion occurs in the patient's presence, was the focus of 18 studies, including 13 medium- or high-quality studies (Table 2). ${ }^{11-14,16-29}$ Patients, attending physicians and resident 


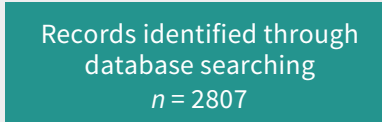
$n=2807$

\section{Additional records identified}

through other sources

$n=145$

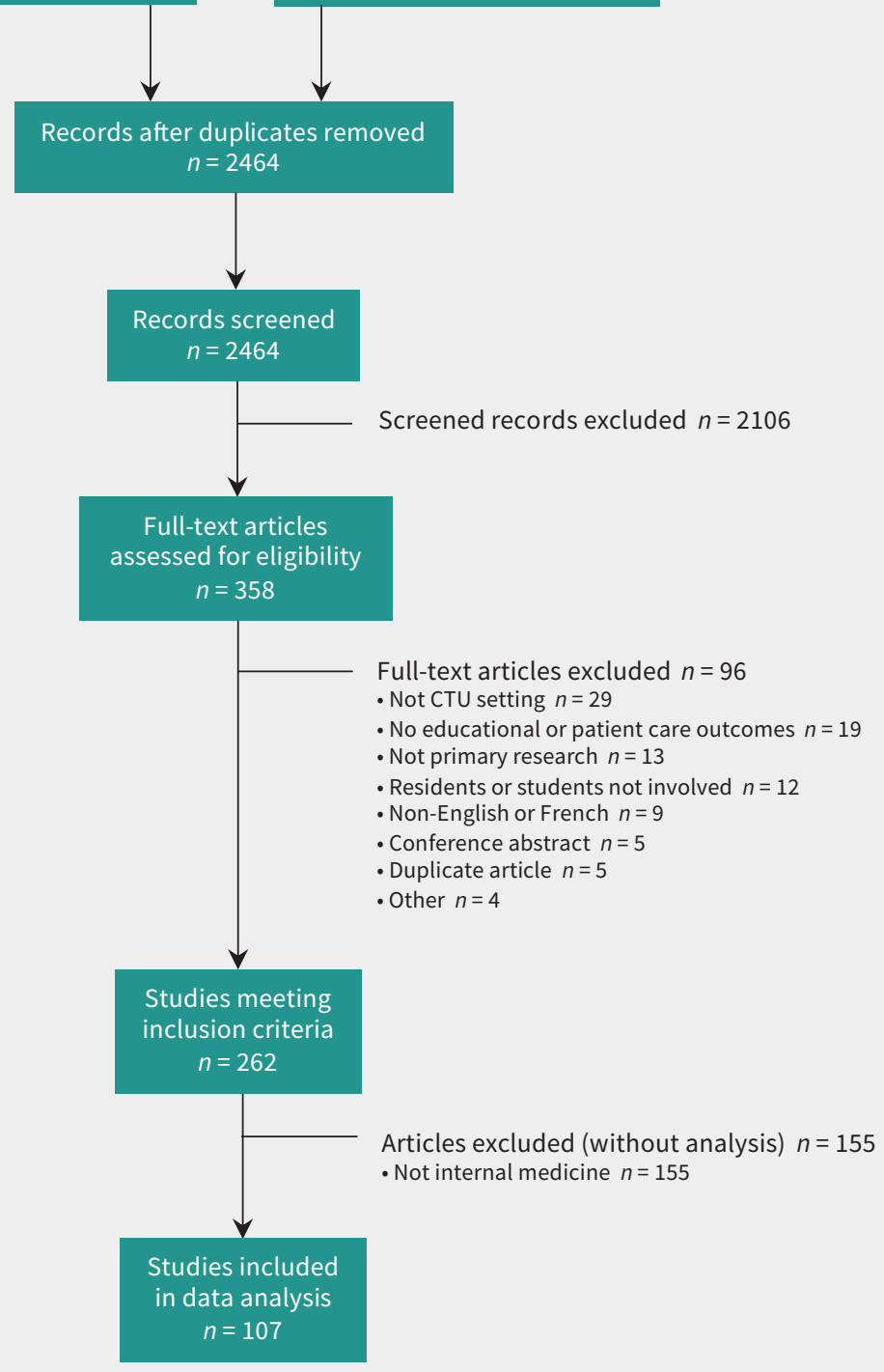

Figure 1: Flow diagram of study selection. Note: CTU = clinical teaching unit.

physicians reported differing perspectives on the value of bedside rounding. In both quantitative and qualitative studies, patients preferred bedside rounds, largely because of a perceived enhancement of patient-physician communication. ${ }^{12,23-25}$ This view is consistent with that of attending physicians, who suggested that bedside rounds are more patient-centred ${ }^{16,20,26}$ and offer an opportunity to model patient-centred communication to trainees. ${ }^{20} \mathrm{How}$ ever, although medical students and residents agreed that patients prefer bedside rounds, ${ }^{24-26}$ they consistently expressed concerns from an educational perspective. Bedside rounds were perceived to take longer or to be inefficient,, ${ }^{12,26,29}$ provide an inferior educational experience ${ }^{12,24,26}$ and lead to reduced autonomy in patient care. ${ }^{12}$ Despite learner perceptions of inefficiency, 3 medium- and high-quality studies reported that structured bedside rounding contributed to either shortened ${ }^{12,14}$ or an unchanged ${ }^{24}$ duration of rounds, although bedside rounds were often introduced in concert with other elements of rounding redesign. ${ }^{12,14}$

Bedside rounds may be more successful when certain implementation principles are followed. Two medium- and highquality studies implementing bedside rounds in combination with other interventions, such as a morning huddle to promote purposeful rounding ${ }^{13}$ or a reduced clinical load, ${ }^{28}$ reported increased overall learner satisfaction. However, the patient census was capped ${ }^{28}$ at 15 or unchanged ${ }^{13}$ at 20 in these studies, and 
Table 1: Characteristics of included studies*

\section{Characteristics}

No. (\%) of studies $n=107$

Geographical setting

North America

Europe

Asia

Oceania

Study design

Survey

Trial (nonrandomized and randomized)

Narrative

Observational

Before and after

$11(10)$

Time series

Semistructured interview

Time motion

$7(7)$

Cohort (prospective or retrospective)

Focus group

Case-control

Field interview

Simulation

Target group

Resident physicians and medical students

Practising physicians

$47(44)$

Patients

Inpatient ward

Other health care professionals

Themes (Star Model) $†$

Strategy

Culture

Structure

Human resources

Information and decision support

Incentives

Study outcomes

Educational

Patient care

Educational and patient care

Study quality

High

Medium

Low

*Most studies involved multiple designs, target groups and foci, so some totals exceed $100 \%$.

†The Star Model is an evidence-based management tool used to guide health system design by analyzing systems according to their related subsystems of strategy, structure, human resources, incentives, information and decision support and culture. ${ }^{10}$ thus the impact of bedside rounds at a higher patient census is unclear. Several studies suggested that more frequent and effective bedside rounds are facilitated by workload factors, such as a reduced clinical load and adequate house staff supports. ${ }^{17,18,26,29}$ In addition, certain patients may be prioritized for bedside rounds, such as those requiring urgent care, newly admitted patients and patients with notable findings on physical examination. ${ }^{19,29}$ We did not identify any studies that evaluated the impact of bedside rounds on direct clinical outcomes such as length of stay or adverse events in CTU settings.

\section{Time allocation}

Eight studies explored how resident physicians allocated their time while working on CTUs, of which 5 were of either medium or high quality (Table 2). ${ }^{30-37}$ Overall, a relatively small amount of time was spent directly with patients (mean $12.9 \%$, range $9.4 \%-17 \%$, $n=6$ studies) ${ }^{30-35}$ or on education (mean $12.8 \%$, range $2 \%-27.2 \%$, $n=4$ studies), ${ }^{31,32,34,35}$ while most residents' time was allocated to computer usage (mean $45.3 \%$, range $40 \%-50.6 \%, n=3$ studies), ${ }^{32,33,35}$ particularly for the purposes of documentation in electronic medical records. ${ }^{33}$

\section{Resource stewardship}

Sixteen studies explored resource stewardship in the CTU setting, of which 14 were either medium or high quality (Table 2)..$^{38-53}$ Most studies introduced an educational ${ }^{39,46-53}$ or process change $\mathrm{e}^{38,39,45}$ intervention, with the goal of improving cost awareness and promoting resource stewardship. Examples of process changes from medium- and high-quality studies included restricting standing laboratory orders, ${ }^{45}$ unbundling order sets ${ }^{38}$ and implementing a novel electronic decision support tool for deprescribing. ${ }^{39}$ Most educational interventions involved didactic lectures or small-group workshops, although 2 medium- and high-quality studies also employed social comparison data, in which individuals received personalized data on their laboratory use relative to their colleagues. ${ }^{48,52}$ Interventions to optimize resource stewardship achieved a variety of outcomes, including a reduction in laboratory use, ${ }^{38,45,47,48,50,52}$ and in prescribing of potentially inappropriate medications, ${ }^{39}$ and measurable cost savings. ${ }^{38,45,48,49,52,53}$ Other studies explored factors related to laboratory use, such as consideration of patient comfort, ${ }^{41}$ methods of tracking test results ${ }^{42}$ and level of clinical training, with more junior trainees tending to order more laboratory tests. ${ }^{44}$

\section{Clinical teaching unit structure}

\section{Interprofessional rounds}

Sixteen studies focused on interprofessional rounds, ${ }^{21,22,54-67}$ of which 9 studies were either medium- or high-quality (Table 3). Reported benefits of this practice included a perceived increase in collaboration $22,62,65$ and communication ${ }^{21,22,57,60,65,66}$ among health care teams; a high-quality time series study also reported earlier discharge times. ${ }^{63}$ Overall, 4 studies assessed structured interprofessional rounds, ${ }^{21,22,61,62}$ which involved regular meetings with the medical team to discuss patient care with nurses, pharmacists and other allied health professionals. Structured interprofessional rounds significantly increased participation of allied health 
Table 2: GRADE-CERQual* summary of analytical theme 1: strategy

CERQual

Summary of review finding

Purposeful rounds, in which certain patients are explicitly prioritized for earlier assessment (e.g., sick patients, potential discharges), may contribute to shorter rounds and improved patient outcomes. ${ }^{11-16}$

Bedside rounds are preferred by patients, but may negatively affect the trainee educational experience, particularly with a higher team workload. ${ }^{11-14,16-29}$

Time allocation of trainees (e.g., spending most time interacting with electronic medical records) may affect the learning experience ${ }^{30-37}$

Educational and process-change interventions can promote resource stewardship in clinical decisionmaking. ${ }^{38-53}$ assessment
High

confidence

confidence

Moderate

confidence

High

confidence

\section{Explanation of CERQual score}

There are moderate concerns regarding relevance. However, there is strong and logical coherence between the intervention and described outcomes.

There are moderate concerns regarding coherence (efficiency of bedside rounds). There are minor concerns regarding relevance.

There are moderate concerns regarding adequacy of data, linking time allocation to trainee experience. There are minor concerns regarding methodology and coherence.

There are minor concerns regarding relevance.
Studies contributing to the review finding

$n=6$

High quality ${ }^{12,14,15}$

Medium quality ${ }^{13,16}$

Low quality ${ }^{11}$

$n=18$

High quality ${ }^{12,14,18-20,24,28,29}$

Medium quality ${ }^{13,16,21,22,25,27}$

Low quality ${ }^{11,17,23,26}$

$n=8$

High quality ${ }^{34,35}$

Medium quality ${ }^{30,33,37}$

Low quality ${ }^{31,32,36}$

$n=16$

High quality ${ }^{39,47-49,53}$

Medium quality ${ }^{38,41,42,44-46,50-52}$

Low quality ${ }^{40,43}$

Note: GRADE-CERQual = Grading of Recommendations Assessment, Development and Evaluation Confidence in the Evidence from Reviews of Qualitative Research

*Comprehensive results from the GRADE-CERQual assessment are reported in Appendix 1, Section 3, Supplementary Table 3.

\section{Table 3: GRADE-CERQual* summary of analytical theme 2: structure}

\section{Summary of review finding}

Interprofessional rounds may improve team collaboration and communication, and enhance discharge planning. ${ }^{21,22,54-67}$

Geographic wards may improve team efficiency and interprofessional collaboration. ${ }^{14,28,56,68}$

"Drip" continuous models of admission reduce daily variability in team workload that, in turn, may improve patient outcomes such as length of stay. ${ }^{69,70}$

Duty hour limits did not affect patient care outcomes, but had variable impacts on trainee experience. ${ }^{72-76}$
CERQual assessment

Moderate confidence

Moderate confidence

Moderate confidence

Moderate confidence
Studies contributing to the review finding

$n=16$

High quality ${ }^{56,63}$

Medium quality ${ }^{21,22,55,61,62,64,65}$ Low quality ${ }^{54,57-60,66,67}$ regarding coherence. However, the overall data suggest a positive impact of interprofessional rounds on discharge planning.

There are moderate concerns regarding adequacy and relevance. However, all studies are medium or high quality and coherent.

There are moderate concerns regarding adequacy. However, all studies are high quality, relevant and coherent.

There are significant concerns regarding coherence and minor concerns regarding adequacy. $n=4$

High quality ${ }^{14,28,56,68}$

$n=2$

High quality ${ }^{69,70}$

$n=5$

High quality ${ }^{72-76}$

Note: GRADE-CERQual = Grading of Recommendations Assessment, Development and Evaluation Confidence in the Evidence from Reviews of Qualitative Research *Comprehensive results from GRADE-CERQual assessment are reported in Appendix 1, Section 3, Supplementary Table 3.

\section{Table 4: GRADE-CERQual* summary of analytical theme 3: human resources}

\section{Summary of review finding}

Limiting clinical workload, such as through a team census cap of 15-20 patients, may improve the trainee educational experience and reduce adverse patient outcomes. ${ }^{11,13,18,28,77-80}$
CERQual assessment

Moderate confidence

\section{Explanation of CERQual score}

There are moderate concerns regarding relevance. However, the data are overal high quality, adequate and coherently supportive of the finding
Studies contributing to the review finding

$n=8$

High quality ${ }^{18,28,77,79,80}$

Medium quality ${ }^{13,78}$

Low quality ${ }^{11}$

Note: GRADE-CERQual = Grading of Recommendations Assessment, Development and Evaluation Confidence in the Evidence from Reviews of Qualitative Research *Comprehensive results from GRADE-CERQual assessment are reported in Appendix 1, Section 3, Supplementary Table 3. 
professionals, ${ }^{22}$ contributed to perceptions of increased patient safety, ${ }^{22,62}$ improved interprofessional communication (especially with nurses $)^{21,22,62}$ and enhanced efficiency, as residents were paged less frequently by nurses. ${ }^{21}$ However, although interprofessional rounds often focused on discharge planning,, ${ }^{59,60,62,63} 2$ highquality randomized trials showed that implementing structured interprofessional rounds did not change average length of stay, ${ }^{62}$ though they significantly reduced rates of adverse events. ${ }^{61}$

\section{Geographic wards}

Four high-quality studies explored geographic wards, in which patients and interprofessional care providers are colocalized in a single physical location, such as a hospital unit or floor, reporting that this may improve team efficiency and interprofessional collaboration (Table 3). ${ }^{14,28,56,68}$ In a time-series study, introduction of geographic wards as part of a multipronged CTU redesign contributed to reduced rounding time and greater nurse attendance on rounds. ${ }^{14}$ Outside of formal rounds, geographic wards also facilitated informal communication events between care providers. ${ }^{56}$ In a quasiexperimental study, a simulation model was used to colocate newly admitted patients with their assigned teams, which resulted in each team interacting with half as many nursing staff, thus improving communication and increasing time for direct patient care for both physicians and nurses. ${ }^{68}$ Although geographic wards were feasible with a low patient census, they were challenging to implement at a higher census without extending wait times in the emergency department. ${ }^{28}$

\section{Models of admission}

Models for admitting new patients were the focus of 2 highquality retrospective cohort studies, which reported that they influence team workload and patient clinical outcomes (Table 3). ${ }^{69,70}$ The first study restructured inpatient teams into a "drip" continuous admission system, such that each team received a small number of daily admissions, moving away from a "bolus" system in which each team admitted a bolus of patients every 4 or 5 days. ${ }^{70}$ The drip model was associated with reduced median length of stay (5.06 v. $4.79 \mathrm{~d}$ ) and less variability in daily discharge rates. The second study found a stepwise increase in patient length of stay, resource use (total costs) and inpatient mortality rates ${ }^{69}$ relative to the total number of patient admissions on their bolus admission day. This suggested that higher trainee workload on admitting days can negatively affect patient outcomes, and potentially lead to harm from the variability in daily workload created by bolus admission systems.

\section{Multi-interventional redesign}

Four studies evaluated multi-interventional initiatives to redesign inpatient CTUs. ${ }^{11,13,28,71}$ Elements found in 3 mediumand high-quality studies included bedside rounding, ${ }^{13,28}$ postdischarge follow-up clinics or rounds, ${ }^{13,28}$ and rounding on patients in a purposeful order. ${ }^{13,71}$ These studies reported a variety of positive outcomes, including improved learner satisfaction ${ }^{13,28,71}$ and reduced length of stay, ${ }^{28,71}$ but their multipronged nature made it difficult to delineate which particular interventions were attributable to observed outcomes. Therefore, we did not conduct a GRADE-CERQual assessment for this study finding.

\section{Duty hours}

Five high-quality studies evaluated the impact of duty hours policies on both educational and patient care outcomes (Table 3). ${ }^{72-76}$ Multiple randomized trials reported no outcome differences when comparing standard duty hour policies and flexible policies that did not specify limits on shift length or time off between shifts. These outcomes included average sleep time per 24 hours, ${ }^{72}$ scores from the American College of Physicians intraining examination, ${ }^{74}$ observed time spent on patient care $^{74}$ and 30-day patient mortality rates. ${ }^{75}$ Two studies reported that stricter duty hours limits (e.g., 16- v. 30-hr limits) increased handovers. ${ }^{73,76}$ Finally, 2 studies, including a randomized trial, reported that trainees in night float or reduced duty hours systems had higher satisfaction. ${ }^{74,76}$ However, in another randomized trial, trainees reported both lower satisfaction and perceived quality of care in reduced duty hour systems. ${ }^{73}$ Overall, in the identified studies, limits on duty hours did not affect patient care outcomes, but variably affected trainees' experiences.

\section{Human resources factors}

\section{Team size and workload}

Team size and workload appeared to influence both educational and patient care outcomes in 8 relevant studies, of which 5 were either medium- or high-quality (Table 4). ${ }^{11,13,18,29,77-80}$ In 2 highquality studies, decreasing the ratio of house staff to attending physicians contributed to more time spent on teaching by attending physicians and an improved educational experience for trainees, without a negative impact on patient care outcomes. ${ }^{28,77}$ In these studies, team composition was modified by either reducing the number of house staff ( 1 attending and 2 or 3 house staff per team $)^{77}$ or by increasing the number of attending physicians ( 2 attendings and 5 house staff per team). ${ }^{28}$

Patient load affects trainee education, team behaviour and patient outcomes. To achieve a reduced clinical workload, 1 high-quality nonrandomized trial implemented a census cap of 15 patients per team and a reduced call frequency for trainees, ${ }^{28}$ finding that interns spent more time engaged in educational activities and endorsed higher satisfaction after intervention. Accordingly, as workload increased from a higher patient census or fewer available house staff, trainees tended to deprioritize teaching in favour of team efficiency ${ }^{78}$ and report that their clinical performance was negatively affected. ${ }^{79}$ In addition, when patient load was higher, teams were less likely to perform bedside rounds ${ }^{18}$ and geographic wards were more difficult to implement. ${ }^{28}$ Finally, in terms of patient outcomes, a high-quality, retrospective cohort study reported that busier teams (> 49 monthly admissions) had a $21 \%$ higher rate of 30 -day readmissions than teams with fewer admissions. ${ }^{80}$

Other studies aimed to enhance inpatient rounding without reducing patient census, through approaches such as morning huddles and bedside rounding. ${ }^{11,13}$ Although trainees reported an improved educational experience, a pre-existing census cap of 20 patients per team was maintained, making it unclear if the intervention would have been successful with a higher patient load. ${ }^{11}$ 


\section{Interpretation}

In this review, we identified several practices that enhanced trainee education or patient care in the CTU setting. Purposeful rounds, interprofessional rounds (specifically structured interprofessional rounds), resource stewardship interventions, drip continuous models of admission and limits on team workload were all practices associated with positive patient care outcomes, including shorter lengths of stay ${ }^{15,69,70}$ and reduced adverse events. ${ }^{15,61,70,80}$ Several practices were found to improve teamwork and communication, ${ }^{14,28,56,60,62}$ including interprofessional rounds and geographic wards. The educational experience of trainees was enhanced when particular teaching behaviours were employed by attending physicians, ${ }^{81-83}$ in several studies involving multi-interventional redesign of the rounding process ${ }^{11,13,28,71}$ and when clinical workload was reduced, such as through team census caps. ${ }^{28,77}$ Bedside rounding was an area of controversy as patients and senior physicians preferred it, ${ }^{12,24,25}$ but residents and medical students did not, ${ }^{12,24,26,29}$ although it may have been better received by trainees with an overall reduced team workload. ${ }^{17,18,26,29}$ Duty hour limits did not measurably affect clinical outcomes in multiple studies, but had variable impacts on the trainee educational experience. ${ }^{72-76}$ Overall, the findings from this review suggest that application of the identified practices may enhance educational value and patient care in CTUs.

Implementing evidence-based practices in medical education is necessary to keep pace with the realities of 21st century health care. In particular, the CTU is an area in which quality improvement and redesign efforts can have a high yield, given that it is a common clinical education model across many countries and clinical specialties. This systematic review explored the CTU through the lens of systems thinking, using the Star Model, to identify design principles that facilitate clinical education and health care delivery. These findings offer insights that are relevant to policies, resource allocation and staffing for teaching hospitals across North America.

Historically, the CTU was designed to provide both an exemplary educational experience and high-quality patient care. ${ }^{3}$ However, a perceived tension between service and learning has since emerged within the CTU, wherein clinical education and patient care may be viewed by trainees as competing activities. ${ }^{84}$ Recognizing this tension, the modern CTU can be viewed as an open system where external contexts interact, including educational priorities, patient care needs, costs of care and health system strain. ${ }^{85}$ Accordingly, although this review focused on factors within the CTU, this perspective emphasizes the importance of considering external forces when designing a CTU. For example, in the context of a growing number of patients who do not require acute hospital care, but who cannot be discharged ${ }^{86}$ the development of nonteaching internal medicine services (e.g., hospitalist teams) may enhance CTUs by concentrating more medically complex patients in teaching teams where their potential for educating learners is maximized. ${ }^{87}$

\section{Limitations}

We focused our search terms primarily on studies in the CTU setting, which may not have captured relevant literature on teaching teams not formally designated as a CTU. Although the narrative synthesis captured valuable mixed-methods data, we had insufficient quantitative evidence to perform a meta-analysis and compare the relative effectiveness of review findings. In addition, most identified themes and included studies identified practices with a potential positive benefit, which may have been influenced by both publication biases and selective outcome reporting. Certain domains of the Star Model framework, such as information and decision support, incentives and culture, are highly relevant to CTUs, but lacked robust data from which we could draw conclusions. As is common in complex systems such as the CTU, it was difficult to discern the specific effects of individual practices in multi-interventional studies. Furthermore, most studies were from single centres, making generalization to other institutions difficult, and many educational outcomes relied on subjective learner impressions or satisfaction. Finally, although we captured evidence from an array of countries, we excluded studies not in English or French, and thus may not have captured articles published in other languages.

\section{Conclusion}

In this systematic review, we used a systems lens to identify several evidence-based practices in internal medicine CTUs that improve clinical education and health care delivery. Understanding and implementing best practice in the CTU is a high-yield area for reform, which aligns with broader efforts to modernize medical education for the 21st century. Targeted, high-quality studies to enhance generalizability and confidence in the identified interventions (e.g., bedside rounds, geographic wards, team census caps) would be ideal areas for future inquiry. Clinical data sets ${ }^{88}$ could be used to quantify how aspects of CTU design affect patient outcomes. Moreover, exploring the external forces that affect the CTU and how these interact with specific interventions, such as hospitalist teams, would complement our findings. Finally, knowledge translation is critical to ensure that best practices in CTU design ultimately become common practice.

\section{References}

1. Creating a community of innovation. Chicago: American Medical Association; 2017.

2. The Future of Medical Education in Canada (FMEC): a collective vision for MD education. Ottawa: The Association of Faculties of Medicine of Canada; 2010.

3. Evans JR, Chute AL, Morley T. The clinical teaching unit as an effective organization for the education of residents under changing medical socio-economic circumstances. I. Objectives and organization of the clinical teaching unit: a white paper. Can Med Assoc J 1966;95:720-7.

4. Moher D, Shamseer L, Clarke M, et al.; PRISMA-P Group. Preferred reporting items for systematic review and meta-analysis protocols (PRISMA-P) 2015 statement. Syst Rev 2015;4:1.

5. Tang B, Sandarage R, Dutkiewicz K, et al. Clinical teaching unit design: a realist systematic review protocol of evidence-based practices for clinical education and health service delivery. BMJ Open 2020;10:e034370.

6. Reed DA, Cook DA, Beckman TJ, et al. Association between funding and quality of published medical education research. JAMA 2007;298:1002-9.

7. Tong A, Sainsbury P, Craig J. Consolidated criteria for reporting qualitative research (COREQ): a 32-item checklist for interviews and focus groups. Int $J$ Qual Health Care 2007;19:349-57.

8. Lewin S, Booth A, Glenton C, et al. Applying GRADE-CERQual to qualitative evidence synthesis findings: introduction to the series. Implement Sci 2018;13(Suppl 1):2.

9. GRADE CERQual [home page]. Available: https://www.cerqual.org/ (accessed 2021 Mar. 29).

10. Golden BR, Martin RL. Aligning the stars: using systems thinking to (re)design Canadian healthcare. Healthc Q 2004;7:34-42. 
11. Reilly JB, Bennett N, Fosnocht K, et al. Redesigning rounds: towards a more purposeful approach to inpatient teaching and learning. Acad Med 2015; 90:450-3.

12. Monash B, Najafi N, Mourad M, et al. Standardized attending rounds to improve the patient experience: a pragmatic cluster randomized controlled trial. J Hosp Med 2017;12:143-9.

13. Bennett NL, Flesch JD, Cronholm P, et al. Bringing rounds back to the patient: a one-year evaluation of the chiefs' service model for inpatient teaching. Acad Med 2017;92:528-36.

14. Huang KTL, Minahan J, Brita-Rossi P, et al. All together now: impact of a regionalization and bedside rounding initiative on the efficiency and inclusiveness of clinical rounds. J Hosp Med 2017;12:150-6.

15. Leykum LK, Chesser H, Lanham HJ, et al. The association between sensemaking during physician team rounds and hospitalized patients' outcomes. J Gen Intern Med 2015;30:1821-7.

16. Najafi N, Monash B, Mourad M, et al. Improving attending rounds: qualitative reflections from multidisciplinary providers. Hosp Pract (1995) 2015;43:186-90.

17. Claridge $A$. What is the educational value of ward rounds? A learner and teacher perspective. Clin Med (Lond) 2011;11:558-62.

18. Gonzalo JD, Wolpaw DR, Lehman E, et al. Patient-centered interprofessional collaborative care: factors associated with bedside interprofessional rounds. J Gen Intern Med 2014;29:1040-7.

19. Gonzalo JD, Heist BS, Duffy BL, et al. The art of bedside rounds: a multi-center qualitative study of strategies used by experienced bedside teachers. J Gen Intern Med 2013;28:412-20.

20. Gonzalo JD, Heist BS, Duffy BL, et al. The value of bedside rounds: a multicenter qualitative study. Teach Learn Med 2013;25:326-33.

21. Schwartz JI, Gonzalez-Colaso R, Gan G, et al. Structured interdisciplinary bedside rounds improve interprofessional communication and workplace efficiency among residents and nurses on an inpatient internal medicine unit. J Interprof Care 2021 Jan. 12 [Epub ahead of print]. doi: 10.1080/ 13561820.2020 .1863932$.

22. Redley B, Campbell D, Stockman K, et al. Mixed methods quality evaluation of structured interprofessional medical ward rounds. Intern Med J 2020;50:222-31.

23. Lehmann LS, Brancati FL, Chen MC, et al. The effect of bedside case presentations on patients' perceptions of their medical care. N Engl J Med 1997; 336:1150-5.

24. Gonzalo JD, Chuang $\mathrm{CH}$, Huang G, et al. The return of bedside rounds: an educational intervention. J Gen Intern Med 2010;25:792-8.

25. Shih AF, Addo-Tabiri NO, Sofair AN. Patients' perceptions of bedside rounding. South Med J 2018;111:281-7.

26. Merchant NB, Federman DG. Bedside rounds valued but not preferred: Perceptions of internal medicine residents and attending physicians in a diverse academic training program. South Med J 2017;110:531-7.

27. Tariq M, Motiwala A, Ali SU, et al. The learners' perspective on internal medicine ward rounds: a cross-sectional study. BMC Med Educ 2010;10:53.

28. McMahon GT, Katz JT, Thorndike ME, et al. Evaluation of a redesign initiative in an internal-medicine residency. N Engl J Med 2010;362:1304-11.

29. Gonzalo JD, Heist BS, Duffy BL, et al. Identifying and overcoming the barriers to bedside rounds: a multicenter qualitative study. Acad Med 2014;89:326-34.

30. D'Souza T, Rosen M, Bertram AK, et al. Use of a real-time location system to understand resident location in an academic medical center. J Grad Med Educ 2019;11:324-7.

31. Guarisco S, Oddone E, Simel D. Time analysis of a general medicine service: results from a random work sampling study. J Gen Intern Med 1994;9:272-7.

32. Fletcher KE, Visotcky AM, Slagle JM, et al. The composition of intern work while on call. J Gen Intern Med 2012;27:1432-7.

33. Mamykina L, Vawdrey DL, Hripcsak G. How do residents spend their shift time? A time and motion study with a particular focus on the use of computers. Acad Med 2016;91:827-32.

34. Chaiyachati KH, Shea JA, Asch DA, et al. Assessment of inpatient time allocation among first-year internal medicine residents using time-motion observations. JAMA Intern Med 2019;179:760-7.

35. Block L, Habicht R, Wu AW, et al. In the wake of the 2003 and 2011 duty hours regulations, how do internal medicine interns spend their time? J Gen Intern Med 2013;28:1042-7.

36. Assis-Hassid S, Grosz BJ, Zimlichman E, et al. Assessing EHR use during hospital morning rounds: a multi-faceted study. PLoS One 2019;14:e0212816.

37. Quinn M, Forman J, Harrod M, et al. Electronic health records, communication, and data sharing: challenges and opportunities for improving the diagnostic process. Diagnosis (Berl) 2019;6:241-8.

38. Attali M, Barel Y, Somin M, et al. A cost-effective method for reducing the volume of laboratory tests in a university-associated teaching hospital. Mt Sinai J Med 2006;73:787-94.
39. McDonald EG, Wu PE, Rashidi B, et al. The MedSafer study: a controlled trial of an electronic decision support tool for deprescribing in acute care. J Am Geriatr Soc 2019;67:1843-50.

40. Sherren C, Day A, Ilan R. Essential and nonessential blood testing in the clinical teaching unit. J Patient Saf 2020;16:307-9.

41. Bodley T, Kwan JL, Matelski J, et al. Self-reported test ordering practices among Canadian internal medicine physicians and trainees: a multicenter cross-sectional survey. BMC Health Serv Res 2019;19:820.

42. Bodley T, Kwan JL, Matelski J, et al. Test result management practices of Canadian internal medicine physicians and trainees. J Gen Intern Med 2019;34:118-24.

43. Moriates C, Soni K, Lai A, et al. The value in the evidence: teaching residents to "choose wisely". JAMA Intern Med 2013;173:308-10.

44. Geleris JD, Shih G, Logio L. Analysis of diagnostic test ordering habits among internal medicine residents. JAMA Intern Med 2018;178:1719-21.

45. McDonald EG, Saleh RR, Lee TC. Mindfulness-based laboratory reduction: reducing utilization through trainee-led daily 'time outs.'. Am J Med 2017;130:e241-4.

46. Post J, Reed D, Halvorsen AJ, et al. Teaching high-value, cost-conscious care: Improving residents' knowledge and attitudes. Am J Med 2013;126:838-42.

47. Miyakis S, Karamanof G, Liontos M, et al. Factors contributing to inappropriate ordering of tests in an academic medical department and the effect of an educational feedback strategy. Postgrad Med J 2006;82:823-9.

48. Ambasta A, Ma IWY, Woo S, et al. Impact of an education and multilevel social comparison-based intervention bundle on use of routine blood tests in hospitalised patients at an academic tertiary care hospital: a controlled preintervention post-intervention study. BMJ Qual Saf 2020;29:1-2.

49. Sommers BD, Desai N, Fiskio J, et al. An educational intervention to improve cost-effective care among medicine housestaff: a randomized controlled trial. Acad Med 2012;87:719-28.

50. Faisal A, Andres K, Rind JAK, et al. Reducing the number of unnecessary rou tine laboratory tests through education of internal medicine residents. Postgrad Med J 2018;94:716-9.

51. Melendez-Rosado J, Thompson KM, Cowdell JC, et al. Reducing unnecessary testing: an intervention to improve resident ordering practices. Postgrad Med J 2017;93:476-9.

52. Minerowicz C, Abel N, Hunter K, et al. Impact of weekly feedback on test ordering patterns. Am J Manag Care 2015;21:763-8.

53. Boushehri E, Khamseh ME, Farshchi A, et al. Effects of morning report case presentation on length of stay and hospitalisation costs. Med Educ 2013;47:711-6.

54. Klobuka AJ, Lee J, Buranosky R, et al. When the reading room meets the team room: resident perspectives from radiology and internal medicine on the effect of personal communication after implementing a resident-led radiology rounds. Curr Probl Diagn Radiol 2019;48:312-22.

55. Nwaesei AS, Jacob BC, Peasah SK, et al. A structured approach to intentional interprofessional experiential education at a non-academic community hospital. Am J Pharm Educ 2019;83:7365.

56. Burm S, Boese K, Faden L, et al. Recognising the importance of informal communication events in improving collaborative care. BMJ Qual Saf 2019;28:289-95.

57. Wilbur K, Teunissen PW, Scheele F, et al. Team member expectations of trainee communicator and collaborator competencies: So shines a good deed in a weary world? Med Teach 2021;43:531-7.

58. Braun B, Grünewald M, Adam-Paffrath R, et al. Impact of interprofessional education for medical and nursing students on the nutritional management of inpatients. GMS J Med Educ 2019;36:Doc11.

59. Vatani H, Sharma H, Azhar K, et al. Required data elements for interprofessional rounds through the lens of multiple professions. J Interprof Care 2020 Nov. 15 [Epub ahead of print]. doi: 10.1080/13561820.2020.1832447.

60. Okoniewska B, Santana MJ, Groshaus H, et al. Barriers to discharge in an acute care medical teaching unit: a qualitative analysis of health providers ' perceptions. J Multidiscip Healthc 2015;8:83-9.

61. O'Leary KJ, Buck R, Fligiel HM, et al. Structured interdisciplinary rounds in a medical teaching unit: improving patient safety. Arch Intern Med 2011;171:678-84.

62. O'Leary KJ, Wayne DB, Haviley C, et al. Improving teamwork: impact of structured interdisciplinary rounds on a medical teaching unit. J Gen Intern Med 2010;25:826-32.

63. Chaiyachati KH, Sofair AN, Schwartz JI, et al. Discharge rounds: implementation of a targeted intervention for improving patient throughput on an inpatient medical teaching service. South Med J 2016;109:313-7.

64. Sang AX, Tisdale RL, Nielsen D, et al. How much time are physicians and nurses spending together at the patient bedside? J Hosp Med 2019;14:468-73.

65. Hamoen EC, van Blankenstein FM, de Jong PGM, et al. Development of a clinical teaching unit in internal medicine to promote interprofessional and multidisciplinary learning: a practical intervention. Teach Learn Med 2021;33:78-88.

66. Allenbaugh J, Spagnoletti CL, Rack L, et al. Health literacy and clear bedside communication: a curricular intervention for internal medicine physicians and medicine nurses. MedEdPORTAL 2019;15:10795. 
67. Ding A, Ratcliffe TA, Diamond A, et al. Ready to collaborate?: medical learner experiences in interprofessional collaborative practice settings. BMC Med Educ 2020;20:85.

68. Bilodeau BL, Stanford DA, Goldszmidt M, et al. Simulated co-location of patients admitted to an inpatient internal medicine teaching unit: potential impacts on efficiency and physician-nurse collaboration. INFOR 2020;58:109-23.

69. Ong M, Bostrom A, Vidyarthi A, et al. House staff team workload and organization effects on patient outcomes in an academic general internal medicine inpatient service. Arch Intern Med 2007;167:47-52.

70. Szecket N, Wong HJ, Wu RC, et al. Implementation of a continuous admission model reduces the length of stay of patients on an internal medicine clinical teaching unit. J Hosp Med 2012; 7:55-9.

71. Southwick F, Lewis M, Treloar D, et al. Applying athletic principles to medical rounds to improve teaching and patient care. Acad Med 2014;89:1018-23.

72. Basner M, Asch DA, Shea JA, et al.; iCOMPARE Research Group. Sleep and alertness in a duty-hour flexibility trial in internal medicine. N Engl J Med 2019; 380:915-23.

73. Desai SV, Feldman L, Brown L, et al. Effect of the 2011 vs 2003 duty hour regulation-compliant models on sleep duration, trainee education, and continuity of patient care among internal medicine house staff: a randomized trial. JAMA Intern Med 2013;173:649-55.

74. Desai SV, Asch DA, Bellini LM, et al. Education outcomes in a duty-hour flexibility trial in internal medicine. N Engl J Med 2018;378:1494-508.

75. Silber JH, Bellini LM, Shea JA, et al. iCOMPARE Research Group. Patient safety outcomes under flexible and standard resident duty-hour rules. N Engl J Med 2019;380:905-14.

76. Mathew R, Gundy S, Ulic D, et al. A reduced duty hours model for senior internal medicine residents: a qualitative analysis of residents' experiences and perceptions. Acad Med 2016;91:1284-92.
77. Spellberg B, Lewis RJ, Sue D, et al. A controlled investigation of optimal internal medicine ward team structure at a teaching hospital. PLoS One 2012;7:e35576.

78. Roy B, Huff N, Estrada C, et al. Contextual influences of trainee characteristics and daily workload on trainee learning preferences. J Hosp Med 2017;12:558-61.

79. Stroud L, Bryden P, Kurabi B, et al. Putting performance in context: the perceived influence of environmental factors on work-based performance. Perspect Med Educ 2015;4:233-43.

80. Averbukh $\mathrm{Y}$, Southern $\mathrm{W}$. The impact of the number of admissions to the inpatient medical teaching team on patient safety outcomes. J Grad Med Educ 2012;4:307-11.

81. Hoellein AR, Feddock CA, Wilson JF, et al. Student involvement on teaching rounds. Acad Med 2007;82:S19-21.

82. Certain LK, Guarino AJ, Greenwald JL. Effective multilevel teaching techniques on attending rounds: a pilot survey and systematic review of the literature. Med Teach 2011;33:e644-50.

83. Merritt FW, Noble MN, Prochazka AV, et al. Attending rounds: What do the allstar teachers do? Med Teach 2017;39:100-4.

84. McCormick I. Designing a CTU [lecture]. The International Conference on Residency Education. 2013 Sept. 26-28; Calgary.

85. Hoffman KG, Donaldson JF. Contextual tensions of the clinical environment and their influence on teaching and learning. Med Educ 2004;38:448-54.

86. Sutherland JM, Crump RT. Alternative level of care: Canada's hospital beds, the evidence and options. Healthc Policy 2013;9:26-34.

87. Abenhaim HA, Kahn SR, Raffoul J, et al. Program description: a hospitalist-run, medical short-stay unit in a teaching hospital. CMAJ 2000;163:1477-80.

88. Verma AA, Guo Y, Kwan JL, et al. Patient characteristics, resource use and outcomes associated with general internal medicine hospital care: the General Medicine Inpatient Initiative (GEMINI) retrospective cohort study. CMAJ Open 2017;5:E842-9.
Competing interests: Brandon Tang reports royalties from Brush Education, outside the submitted work. No other competing interests were declared.

This article has been peer reviewed.

Affiliations: Department of Medicine (Tang, Hatala); Faculty of Medicine (Sandarage, Chai, Dawson); Divisions of General Internal Medicine (Dutkiewicz, McCormick, Kassen), Infectious Diseases (Saad) and Community Internal Medicine (Kassen); Vancouver Fraser Medical Program and Woodward Library (Kitchin); University of British Columbia, Vancouver, BC

Contributors: Brandon Tang, Katrina Rose Dutkiewicz, Stephan Saad, Rose Hatala, Iain McCormick and Barry Kassen contributed to the conception and design of the work. Brandon Tang, Ryan Sandarage, Jocelyn Chai, Kristin Dawson, Katrina Dutkiewicz and Stephan Saad contributed to data acquisition, analysis and interpretation. Brandon Tang and Ryan Sandarage drafted the manuscript. All of the authors revised it critically for important intellectual content, gave final approval of the version to be published and agreed to be accountable for all aspects of the work.

Content licence: This is an Open Access article distributed in accordance with the terms of the Creative Commons Attribution (CC BY-NC-ND 4.0) licence, which permits use, distribution and reproduction in any medium, provided that the original publication is properly cited, the use is noncommercial (i.e., research or educational use), and no modifications or adaptations are made. See: https://creativecommons. org/licenses/by-nc-nd/4.0/

Funding: This work was supported by the Division of General Internal Medicine, University of British Columbia, Vancouver, BC.

Data sharing: A list of studies meeting inclusion criteria but not included in our analysis (i.e., studies in specialties other than internal medicine) may be available upon written request to the corresponding author.

Accepted: Nov. 19, 2021

Correspondence to: Brandon Tang, bran.tang@mail.utoronto.ca 